\title{
Interproximal grooving of lower second molars in WLH 4
}

\author{
Arthur C. Durband ${ }^{1,2,3}$, Michael C. Westaway ${ }^{4}$ and Daniel R.T. Rayner ${ }^{5}$
}

\begin{abstract}
Interproximal grooving of the teeth is a form of nonmasticatory wear commonly found in precontemporary human populations. While its cause is debated, it is thought to be due to the repeated abrasion of fibrous materials across the distal surfaces of teeth during processing. This report describes the presence of interproximal grooves on the distal surfaces of the lower second molars of the WLH 4 individual from the Willandra Lakes in New South Wales, Australia. Although there has been considerable discussion regarding the distinct occlusal wear pattern in WLH 3, this is the first recorded instance of an interproximal wear pattern in the Willandra Lakes region, and has implications for our understanding of cultural behaviors practiced by those populations in the late Holocene.
\end{abstract}

\section{Introduction}

Dental remains and dental wear patterns can provide important information for understanding human behavior. These data are often used to inform researchers about dietary choices, but dental wear caused by non-masticatory functions is equally important for elucidating other forms of habitual behavior. Interproximal grooves are one type of wear caused by non-masticatory use of the teeth to perform specific tasks. These features have been reported in various pre-contact populations, including Native Americans (Berryman et al. 1979; Schulz 1977; Ubelaker et al. 1969), Bushmen from South Africa (Wallace 1974), prehistoric Pakistani groups (Lukacs and Pastor 1988) and even the Krapina Neandertals (Frayer and Russell 1987). Likewise, in Australia, interproximal grooving of the teeth has been documented in skeletal samples (e.g. Brown and Molnar 1990; Campbell 1925).

Interproximal grooves are most commonly found on the distal sides of premolars and molars, are oriented horizontally, are semicircular in cross-section, and can be from 1-4 mm in diameter (Brown and Molnar 1990). The aetiology of interproximal grooving has been much discussed in the literature (see Brown and Molnar 1990; Lukacs and Pastor 1988). In the Swanport (South Australia) population these marks appear on $41 \%$ of individuals, with the frequency in males twice that of females (Brown and Molnar 1990). Brown and Molnar (1990) cited ethnographic films, such as The Woomera (Campbell 1958) and Aboriginal Spears (Campbell 1963), that depict Aboriginal men dragging sinews through their clenched posterior teeth for the purpose of stripping them, hypothesising that the grooves were most likely caused by the preparation of animal sinews for uses such as making spears or woomeras. Sinews or other fibres worked in this way

${ }^{1}$ Department of Sociology, Anthropology and Social Work, Texas Tech University, Lubbock Texas 79409-1012, USA $<$ arthur.durband@ttu.edu>

2 Department of Archaeology, Flinders University, Adelaide SA 5001, Australia

${ }^{3}$ Department of Anthropology, Science Centre, South Australian Museum, Adelaide SA 5001, Australia

${ }^{4}$ Cultural Environments Program, Queensland Museum, South Bank Qld 4001, Australia <michael.westaway@qm.qld.gov.au>

${ }^{5}$ School of Archaeology and Anthropology, The Australian National University, Canberra ACT 0200, Australia <daniel.rayner@anu.edu.au> would easily slip into the interproximal spaces between teeth, and would be extricated by dragging those materials laterally (Brown and Molnar 1990:550):

... [the craftsman chews the sinew] until it is soft and pliable. The sinew, which at this stage appears to be a few millimeters in width, is then drawn between the occluded teeth from left to right side with the right hand while the left hand holds the trailing end of the sinew, guiding its passage through the teeth. The action is then repeated and reversed....

This method of working animal sinews or some other flexible fibrous material for tool production provides a reasonable explanation for the production of these horizontally oriented interproximal grooves in precontemporary Australian groups.

In this paper we report the appearance of interproximal grooving on the lower second molars of the WLH 4 individual from the Willandra Lakes region of New South Wales (NSW). Partially described by Webb (1989), this skeleton is presently undated, though it is clearly considerably more recent than other Willandra Lakes skeletal remains. Despite the more recent age of this burial, however, we feel it is likely that this individual is either of pre-contact age or dates to around the time of early European contact. Thus, these remains provide an important insight into the biology and cultural practices of pre-contact Australian groups in the region.

\section{Materials}

The WLH 4 remains were excavated by Wilfred Shawcross in 1974 at the Walls of China 3 site in the same season that the Mungo $\mathrm{B}$ excavation was conducted. It is unique in the Willandra series in many ways: unlike the majority of fossilised remains the bone is not heavily mineralised; unlike the majority of now eroded burials situated on the surface, WLH 4 was a carefully excavated burial; and it is also represented by most of its skeletal elements. The individual has also been claimed to have preserved aDNA (Adcock et al. 2001), which further supports a more recent date for the burial. Its mitochondrial sequence fits within the known mitochondrial haplotype range of modern Australian populations (Adcock et al. 2001). The WLH 4 remains were studied by the authors during a general assessment and analysis of the Willandra Lakes skeletal collection, at which time the grooves were noted.

\section{Results and Discussion}

WLH 4 is characterised by heavy wear apparent on all preserved teeth; this may be due to a combination of an abrasive diet with some degree of non-masticatory use of the teeth. In addition to the extreme occlusal wear in the teeth of WLH 4 there are grooves that appear bilaterally on the lower second molars. These grooves are horizontally oriented and are approximately $1.5-2 \mathrm{~mm}$ in height (Figures 1-3). Owing to the extensive wear to the molar crowns, it is difficult to determine exactly where these grooves occur in relation to the crown, but their positioning is approximately at the level of the cemento-enamel junction. This 
is a common location for these types of features (Brown and Molnar 1990; Formicola 1988; Ubelaker et al. 1969). The grooves lie directly opposite one another, making it likely that they were formed simultaneously through the repeated dragging of some fibrous material across those surfaces of the teeth.

To our knowledge this is the first published observation of this type of wear pattern in an individual from the Willandra Lakes. It provides insights into the types of activities being performed by individuals in the Willandra, and likewise extends the known area for this form of material processing in Australia. These exact grooves have not been seen in the (admittedly limited) Pleistocene dental sample from the Willandra, which could indicate an evolution of cultural activities in the region. The only other individual within the Willandra series that exhibits any level of significant dental attrition is WLH 3 (Mungo Man), described in some detail by Webb (1995:60). His left first and second molars exhibit extreme wear, which Webb (1995) considered similar to that seen elsewhere in Holocene populations from the Murray. Microscopic examination revealed a series of striations or parallel grooves cut into both the exposed dentine and the remaining enamel, and this was interpreted as a wear pattern associated with material processing rather than food processing (Webb 1995:61). We would concur with this interpretation, and note that it indicates evidence within the skeletal series of the antiquity of teeth use for non-masticatory processes spanning a period of 42,000 years.

The Willandra is a highly eroding landscape and archaeological materials, including burials, are frequently eroding out of the system. The Garnpung-Leaghur interlake is perhaps one of the richest parts of the system for the erosion of burials. An active program of rescue archaeology now exists in the Willandra Lakes as a result of recent ARC grants, and much valuable archaeological information is now being recorded as a result. If recording dental wear in exposed remains prior to reburial could be added to the Aboriginal Cultural Officers' site recording toolkit, it is likely that further insights into continuity and change in processing material culture could be learned.

\section{Acknowledgements}

The authors would like to thank the Elders of the Traditional Tribal Groups of the Willandra Lakes for their kind permission to study these remains. The late Alan Thorne provided logistical support for this project. Wilfred Shawcross shared details of the excavation. Leanne Mitchell and David Lambert assisted with the photographs. This work was supported by the Franklin Grant program of the American Philosophical Society and Texas Tech University.

\section{References}

Adcock, G.J., E.S. Dennis, S. Easteal, G.A. Huttley, L.S. Jermiin, W.J. Peacock and

A. Thorne 2001 Mitochondrial DNA sequences in ancient Australians: Implications for modern human origins. Proceedings of the National Academy of Sciences 98:537-542.

Berryman, H.E., D.W. Owsley and A.M. Henderson 1979 Non-carious interproximal grooves in Arikara Indian dentitions. American Journal of Physical Anthropology 50:209-212.

Brown, T. and S. Molnar 1990 Interproximal grooving and task activity in Australia.

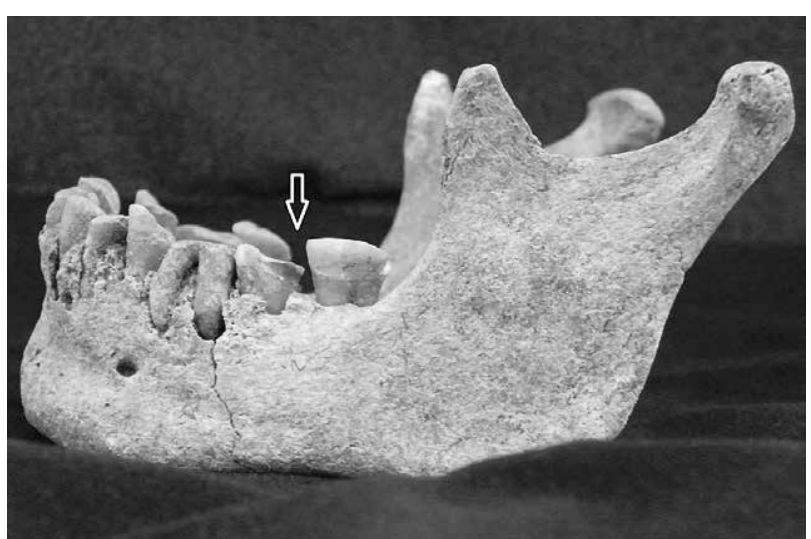

Figure 1 Left lateral view of the WLH 4 mandible. The white arrow indicates the position of the interproximal groove on the second molar.

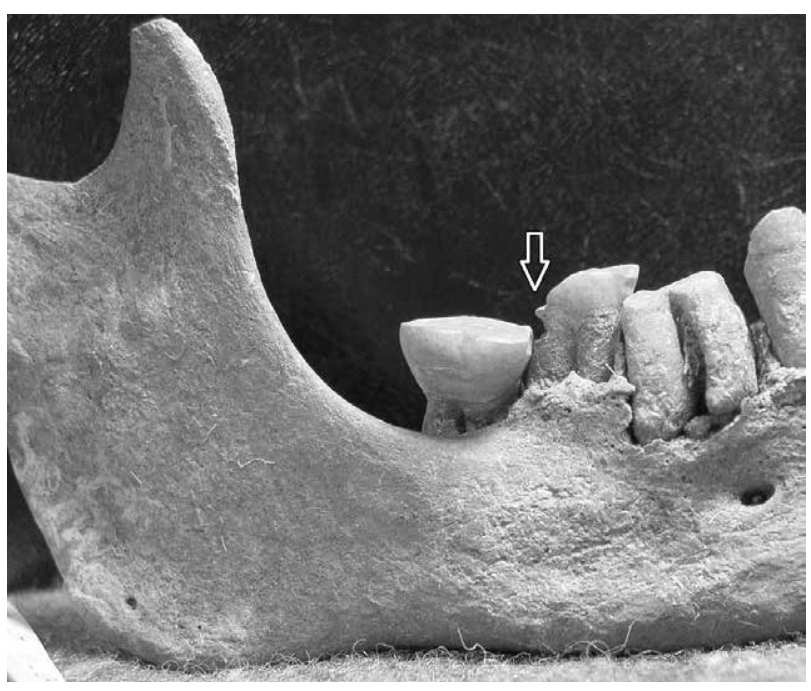

Figure 2 Right lateral view of the WLH 4 mandible. The white arrow indicates the position of the interproximal groove on the second molar.

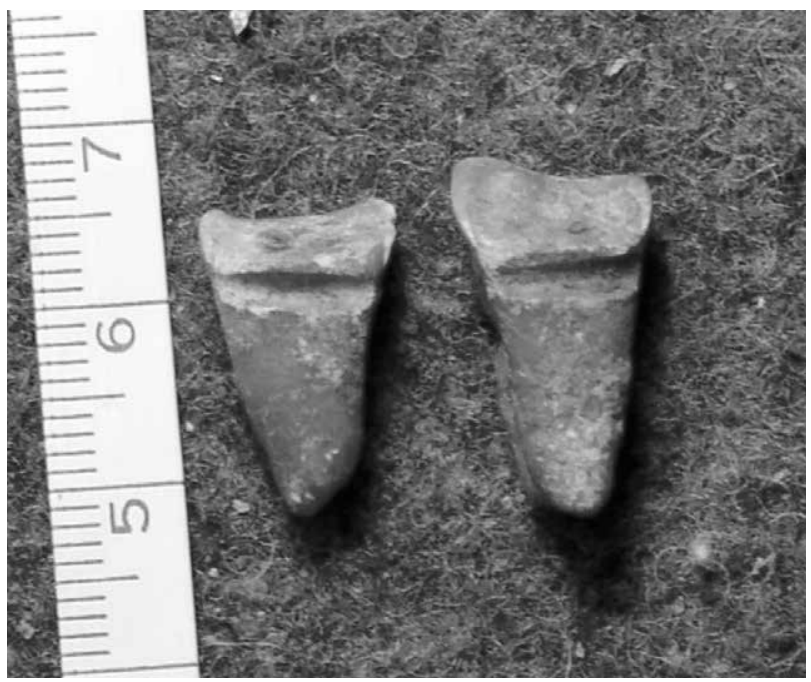

Figure 3 Distal surfaces of both second molars of WLH 4.

American Journal of Physical Anthropology 81:545-553.

Campbell, T.D. 1925 Dentition and Palate of the Australian Aboriginal. Adelaide: The Hassell Press.

Campbell, T.D. 1958 The Woomera. Adelaide: Board of Aboriginal Studies. Documentary film.

Campbell, T.D. 1963 Aboriginal Spears. Adelaide: Board of Aboriginal Studies. 
Documentary film.

Formicola, V. 1988 Interproximal grooving of teeth: Additional evidence and interpretation. Current Anthropology 29(4):663-671.

Frayer, D.W. and M.D. Russell 1987 Artificial grooves on the Krapina Neanderthal teeth. American Journal of Physical Anthropology 74:393-405.

Lukacs, J.R. and R.F. Pastor 1988 Activity-induced patterns of dental abrasion in prehistoric Pakistan: Evidence from Mehrgarh and Harappa. American Journal of Physical Anthropology 76:377-398.

Schulz, P.D. 1977 Task activity and anterior tooth grooving in prehistoric California Indians. American Journal of Physical Anthropology 46:87-92.

Ubelaker D.H., T.W. Phenice and W.M. Bass 1969 Artificial interproximal grooving of the teeth in American Indians. American Journal of Physical Anthropology 30:145-149.

Wallace, J.A. 1974 Approximal grooving of teeth. American Journal of Physical Anthropology 40:385-390.

Webb, S.G. 1989 The Willandra Lakes Hominids. Canberra: The Australian National University.

Webb, S.G. 1995 Palaeopathology of Australian Aborigines. Cambridge: Cambridge University Press. 